\title{
Precision predictions for electroweak superpartner production at hadron colliders with RESUMMINO
}

\author{
Benjamin Fuks $^{1,2, a}$, Michael Klasen ${ }^{3}$, David R. Lamprea ${ }^{3}$, Marcel Rothering ${ }^{3}$ \\ ${ }^{1}$ Theory Division, Physics Department, CERN, 1211 Geneva 23, Switzerland \\ ${ }^{2}$ Institut Pluridisciplinaire Hubert Curien/Département Recherches Subatomiques, Université de Strasbourg/CNRS-IN2P3, 23 Rue du Loess, \\ 67037 Strasbourg, France \\ ${ }^{3}$ Institut für Theoretische Physik, Westfälische Wilhelms-Universität Münster, Wilhelm-Klemm-Straße 9, 48149 Münster, Germany
}

Received: 5 April 2013 / Revised: 16 May 2013 / Published online: 2 July 2013

(C) The Author(s) 2013. This article is published with open access at Springerlink.com

\begin{abstract}
We describe the Resummino package, a C++ and FORTRAN program dedicated to precision calculations in the framework of gaugino and slepton pair production at hadron colliders. This code allows to calculate transversemomentum and invariant-mass distributions as well as total cross sections by combining the next-to-leading order predictions obtained by means of perturbative QCD with the resummation of the large logarithmic contributions arising in the small transverse-momentum region and close to the production threshold. The results computed in this way benefit from reduced theoretical uncertainties, compared to a pure next-to-leading order approach as currently employed in the experimental analyses searching for sleptons and gauginos at hadron colliders. This is illustrated by using RESUMMINO in the context of a typical supersymmetric benchmark point dedicated to superpartner searches at the Large Hadron Collider.
\end{abstract}

\section{Introduction}

Over the last forty years, theoretical developments and experimental discoveries in high-energy physics have led to an extremely coherent picture, the so-called Standard Model of particle physics. In particular, the recent observation of a neutral bosonic particle compatible with a Standard-Modellike Higgs boson $[1,2]$ represents an impressive success of this theoretical framework. However, many questions, such as the stabilization of the mass of a fundamental scalar particle with respect to radiative corrections, remain unanswered. Consequently, the Standard Model is widely accepted as an effective theory implied by a more fundamental one. Among

a e-mail: benjamin.fuks@iphc.cnrs.fr the plethora of candidates for this new physics theory, weakscale supersymmetry $[3,4]$ is one of the most appealing and studied options. It not only addresses the above-mentioned hierarchy problem but also provides a solution for the unification of the gauge couplings at high energies and explains the presence of dark matter in the Universe.

Experimental searches, especially at the Large Hadron Collider (LHC) at CERN, for the supersymmetric partners of the Standard Model particles are therefore among the main items of the experimental program in high-energy physics. Up to now, both the ATLAS and CMS collaborations have mainly focused on seeking for hints of squarks and gluinos, being strongly produced. All results are however compatible with the Standard Model expectation [5, 6]. As a consequence, limits on the masses of the squarks and gluino are pushed to higher and higher scales and the experimental attention starts to shift towards the pair production of the electroweak slepton, neutralino and chargino eigenstates.

Investigations at a center-of-mass energy of $8 \mathrm{TeV}$ of the trilepton golden signature have already led to bounds of several hundreds of $\mathrm{GeV}$ on the masses of these particles $[7,8]$. However, in contrast to the strong production channels where estimates of signal cross sections rely on precise theoretical predictions at the next-to-leading order and next-to-leading logarithmic accuracy [9-15], searches for the weak superpartners are only based on next-to-leading order computations, suffering from larger theoretical uncertainties [16-18].

For an efficient suppression of the Standard Model background and a more precise extraction of the underlying supersymmetric mass limits (or a measurement of the supersymmetric parameters in the case of a discovery), accurate theoretical calculations of signal cross sections and key kinematical distributions are imperative. Along these 
lines, while supersymmetric particle pairs are produced with a vanishing transverse momentum $\left(p_{T}\right)$ at the lowest order in the strong coupling $\alpha_{s}$, gluon radiation from quark-antiquark initial states and their splitting into quarkantiquark pairs at $\mathcal{O}\left(\alpha_{s}\right)$ induce transverse momenta extending to larger values. Next-to-leading order corrections have therefore to be taken into account. However, the perturbative calculation diverges at small $p_{T}$, which indicates the need for soft-gluon resummation to all orders and for a consistent matching with the fixed order results. On the same footings, when supersymmetric particle pairs are produced close to the production threshold, soft gluon emission again leads to large logarithmic terms that must be resummed and matched to fixed order in order to obtain reliable predictions.

These considerations motivate us to introduce in this work the computer code RESUMMINO, implemented in $\mathrm{C}++$ and FORTRAN and that can be downloaded from the website

http: / / www.resummino.org

It combines a leading order calculation of slepton and gaugino pair production at hadron colliders [19-21] supplemented by the associated supersymmetric QCD next-toleading order corrections $[17,18]$, with the resummation of the leading and next-to-leading logarithms to all orders in the threshold $[17,18,22]$ and small transverse-momentum [22-24] regimes or simultaneously in both $[22,25,26]$.

In Sect. 2 of this paper, we briefly review the three resummation formalisms included in the RESUMMINO package, giving the explicit form of the implemented formulas, as well as the techniques employed to calculate the underlying integrals. Section 3 is more technical and is dedicated to the installation of the program and its running. A set of illustrative results are then shown in Sect. 4, after choosing a benchmark point typical for supersymmetry searches at the LHC.

\section{Resummation formalisms}

\subsection{Main features}

In perturbative $\mathrm{QCD}$, the doubly-differential cross section describing the production, at hadron colliders, of a gaugino or a slepton pair with an invariant mass $M$ and a transverse momentum $p_{T}$ is calculated by means of the QCD factorization theorem. The fixed order result is in this way obtained by convolving the partonic cross section $\mathrm{d} \sigma_{a b}$, computed at a given order in the strong coupling, with the universal densities $f_{a}$ and $f_{b}$ of the partons $a$ and $b$ carrying the momentum fractions $x_{a}$ and $x_{b}$ of the colliding hadrons,

$$
M^{2} \frac{\mathrm{d}^{2} \sigma}{\mathrm{d} M^{2} \mathrm{~d} p_{T}^{2}}(\tau)
$$

$$
\begin{aligned}
= & \sum_{a b} \int_{0}^{1} \mathrm{~d} x_{a} \mathrm{~d} x_{b} \mathrm{~d} z\left[x_{a} f_{a}\left(x_{a}, \mu_{F}^{2}\right)\right] \\
& \times\left[x_{b} f_{b}\left(x_{b}, \mu_{F}^{2}\right)\right]\left[z \mathrm{~d} \sigma_{a b}\left(z, M^{2}, p_{T}^{2}, \mu_{F}^{2}, \mu_{R}^{2}\right)\right] \\
& \times \delta\left(\tau-x_{a} x_{b} z\right) .
\end{aligned}
$$

In this expression, the unphysical factorization and renormalization scales are respectively denoted by $\mu_{F}$ and $\mu_{R}$ and we have introduced the quantity $\tau=M^{2} / S_{h}, S_{h}$ standing for the hadronic center-of-mass energy. After performing a Mellin transform with respect to the variable $\tau$, this cross section can be reexpressed as a simple product of parton densities and the partonic cross section in the conjugate Mellin $N$-space,

$$
\begin{aligned}
& M^{2} \frac{\mathrm{d}^{2} \sigma}{\mathrm{d} M^{2} \mathrm{~d} p_{T}^{2}}(N-1) \\
& =\sum_{a b} f_{a}\left(N, \mu_{F}^{2}\right) f_{b}\left(N, \mu_{F}^{2}\right) \\
& \quad \times \sigma_{a b}\left(N, M^{2}, p_{T}^{2}, \mu_{F}^{2}, \mu_{R}^{2}\right),
\end{aligned}
$$

where the Mellin moments of the quantities $F=\sigma, \sigma_{a b}, f_{a}$ and $f_{b}$ are defined by

$F(N)=\int_{0}^{1} \mathrm{~d} y y^{N-1} F(y)$,

with $y=\tau, z, x_{a}$ and $x_{b}$, respectively. Under the form of Eq. (2), it is possible to effectively resum to all orders in $\alpha_{s}$ the large logarithmic terms arising in the critical regions, i.e., when the transverse momentum tends towards zero and/or when the partonic center-of-mass energy is close to the production threshold. The exact form of the resummed cross sections is detailed for the small transverse momentum, threshold and joint regimes in Sect. 2.2, Sect. 2.3 and Sect. 2.4, respectively.

Although these large logarithms must clearly be resummed in the critical regions, the full perturbative computation, only partially accounted for by resummation, is expected to be reliable away from these regions. In order to obtain valid predictions in all kinematical regions, the fixed $\operatorname{order}\left(\sigma^{(\text {f.o. })}\right)$ and resummed $\left(\sigma^{(\text {res. })}\right)$ results have then to be consistently combined by subtracting from their sum their overlap $\left(\sigma^{\text {(exp.) }}\right)$,

$\sigma_{a b}=\sigma_{a b}^{\text {(res.) }}+\sigma_{a b}^{\text {(f.o.) }}-\sigma_{a b}^{\text {(exp.) }}$.

The latter is obtained by expanding the resummation formula to the desired accuracy, i.e., at $\mathcal{O}\left(\alpha_{s}\right)$ in our case, and is thus dependent on the employed resummation formalism. The analytical expressions of $\sigma^{\text {(exp.) }}$ are therefore given in the relevant subsections below. 
While the fixed order result $\sigma^{\text {(f.o.) }}$ can in general be directly computed in physical space, or in other words by directly using Eq. (1), the resummed component and the calculation of its expansion at a given order in $\alpha_{s}$ requires factorization properties that only hold in conjugate spaces so that Eq. (2) is employed. An inverse Mellin transform is consequently necessary to get back to the physical space, which implies to take care of the singularities possibly arising at the level of the $N$-space cross section. This is achieved by choosing an integration contour inspired by the principal value procedure and minimal prescription [27, 28]. On this contour, the Mellin variable $N$ is parameterized as a function of two parameters $C$ and $\phi$ and one variable $y$,

$$
N(y)=C+y e^{ \pm i \phi} \quad \text { with } y \in[0, \infty[.
$$

The parameter $C$ is chosen such that, on the one hand, the poles in the Mellin moments of the parton densities related to the Regge singularity lie to the left of the integration contour and that, on the other hand, the Landau pole related to the running of the strong coupling constant lies to its right. Furthermore, the phase $\phi$ can formally be chosen anywhere in the range $[\pi / 2, \pi[$.

\subsection{Threshold resummation at the next-to-leading logarithmic accuracy}

In the threshold regime, the slepton or gaugino pair invariant mass $M^{2}$ is close to the partonic center-of-mass energy or equivalently, the Mellin variable $N$ tends towards infinity. In this case, refactorization allows us to rewrite the partonic cross section $\sigma_{a b}$ obtained after integrating Eq. (2) over the transverse momentum $p_{T}$ into a closed exponential form [29-34],

$$
\begin{aligned}
& \sigma_{a b}^{(\text {res. })}\left(N, M^{2}, \mu_{F}^{2}, \mu_{R}^{2}\right) \\
&= \mathcal{H}_{a b}\left(M^{2}, \mu_{F}^{2}, \mu_{R}^{2}\right) \\
& \quad \times \exp \left[\mathcal{G}_{a b}\left(N, M^{2}, \mu_{F}^{2}, \mu_{R}^{2}\right)\right] .
\end{aligned}
$$

The hard part of the scattering process, independent of the Mellin variable $N$, is embedded within the perturbatively computable function $\mathcal{H}_{a b}$. On different footings, the function $\mathcal{G}_{a b}$, that can also be computed by means of perturbation theory, collects soft and collinear parton radiation and absorbs the large logarithmic contributions arising at fixed order.

It has however recently been shown that Eq. (6) can be improved, at the next-to-leading logarithmic accuracy, by including and resumming subleading terms stemming from universal collinear radiation of the initial state partons [3538]. This improvement procedure leads to a resummed cross section explicitly depending on the one-loop approximation of the QCD evolution operator $E_{a b}^{(1)}$, which drives the behavior of the parton-into-parton density functions with the energy and encompasses collinear radiation [39]. Consequently, the original resummation formula is modified to

$$
\begin{aligned}
& \sigma_{a b}^{(\text {res. })}\left(N, M^{2}, \mu_{F}^{2}, \mu_{R}^{2}\right) \\
& =\sum_{a^{\prime}, b^{\prime}} E_{a a^{\prime}}^{(1)}\left(N, M^{2} / \bar{N}^{2}, \mu_{F}^{2}\right) \\
& \quad \times E_{b b^{\prime}}^{(1)}\left(N, M^{2} / \bar{N}^{2}, \mu_{F}^{2}\right) \tilde{\mathcal{H}}_{a^{\prime} b^{\prime}}\left(M^{2}, \mu_{R}^{2}\right) \\
& \quad \times \exp \left[\tilde{\mathcal{G}}_{a^{\prime} b^{\prime}}\left(\bar{N}, M^{2}, \mu_{R}^{2}\right)\right],
\end{aligned}
$$

where we have introduced the reduced Mellin variable defined by $\bar{N}=N e^{\gamma_{E}}$. The improved soft and hard functions $\tilde{\mathcal{G}}_{a b}$ and $\tilde{\mathcal{H}}_{a b}$ can still be perturbatively computed and read, at the next-to-leading logarithmic accuracy,

$$
\begin{aligned}
& \tilde{\mathcal{H}}_{a b}\left(M^{2}, \mu_{R}^{2}\right) \\
& \quad=\tilde{\mathcal{H}}_{a b}^{(0)}\left(M^{2}, \mu_{R}^{2}\right)+\frac{\alpha_{s}\left(\mu_{R}^{2}\right)}{2 \pi} \tilde{\mathcal{H}}_{a b}^{(1)}\left(M^{2}, \mu_{R}^{2}\right), \\
& \tilde{\mathcal{G}}_{a b}\left(N, M^{2}, \mu_{R}^{2}\right) \\
& \quad=\tilde{g}_{a b}^{(1)}\left(\frac{\alpha_{s}}{2 \pi} \beta_{0} \ln \bar{N}\right) \ln \bar{N}+\tilde{g}_{a b}^{(2)}\left(\frac{\alpha_{s}}{2 \pi} \beta_{0} \ln \bar{N}, \frac{M^{2}}{\mu_{R}^{2}}\right) .
\end{aligned}
$$

The arguments of the leading and next-to-leading contributions to the Sudakov form factor $\tilde{\mathcal{G}}_{a b}$ depend, in addition to the reduced Mellin variable, on the one-loop coefficient of the QCD beta-function $\beta_{0}$. This quantity and the associated two-loop coefficient $\beta_{1}$ given for further references are defined, in our normalization conventions, by

$$
\begin{aligned}
& \beta_{0}=\frac{11}{6} C_{A}-\frac{2}{3} N_{f} \tau_{R}, \\
& \beta_{1}=\frac{1}{6}\left[17 C_{A}^{2}-5 C_{A} N_{f}-3 C_{F} N_{f}\right],
\end{aligned}
$$

for $N_{f}$ active quark flavors. In those expressions, the group theory invariants of $S U(3)$ are standard and read $C_{A}=3$, $C_{F}=4 / 3$ and $\tau_{R}=1 / 2$.

The first two coefficients $\tilde{g}_{a b}^{(1)}$ and $\tilde{g}_{a b}^{(2)}$ of the function $\tilde{\mathcal{G}}_{a b}$ allow to resum the leading and next-to-leading logarithmic contributions yielded by soft and collinear radiation. In the $\overline{\mathrm{MS}}$ renormalization scheme, their functional form is explicitly given by [17, 18, 29-31, 35]

$$
\begin{aligned}
& \tilde{g}_{a b}^{(1)}(\lambda)=\frac{1}{2 \lambda \beta_{0}}\left(A_{a}^{(1)}+A_{b}^{(1)}\right)(2 \lambda+\ln (1-2 \lambda)), \\
& \tilde{g}_{a b}^{(2)}\left(\lambda, \frac{M^{2}}{\mu_{R}^{2}}\right) \\
& =-\frac{1}{2 \beta_{0}^{2}}\left[A_{a}^{(2)}+A_{b}^{(2)}\right][2 \lambda+\ln (1-2 \lambda)]
\end{aligned}
$$




$$
\begin{aligned}
& +\frac{1}{\beta_{0}}\left[B_{a}^{(1)}+B_{b}^{(1)}\right] \ln (1-2 \lambda) \\
& +\frac{1}{2 \beta_{0}}\left[A_{a}^{(1)}+A_{b}^{(1)}\right][2 \lambda+\ln (1-2 \lambda)] \ln \frac{M^{2}}{\mu_{R}^{2}} \\
& +\frac{\beta_{1}}{2 \beta_{0}^{3}}\left[A_{a}^{(1)}+A_{b}^{(1)}\right][2 \lambda+\ln (1-2 \lambda) \\
& \left.+\frac{1}{2} \ln ^{2}(1-2 \lambda)\right] .
\end{aligned}
$$

This shows that the first two terms of the perturbative expansion of the Sudakov form factor only depend on the $A_{a}$ and $B_{a}$ resummation functions respectively describing soft and collinear radiation and flavor-conserving collinear radiation. The leading terms of their expansion as series in powers of $\alpha_{s}$ have been calculated in the past and are given by [30, 40, 41]

$$
A_{a}^{(1)}=2 C_{a}, \quad A_{a}^{(2)}=2 C_{a}\left[\left(\frac{67}{18}-\frac{\pi^{2}}{6}\right) C_{A}-\frac{5}{9} N_{f}\right],
$$

and

$B_{q}^{(1)}=-3 C_{F} \quad$ and $\quad B_{g}^{(1)}=-2 \beta_{0}$,

for the $A_{a}$ and $B_{a}$ functions.

Turning to the hard parts of the resummed cross section, the leading and next-to-leading order contributions to the $\tilde{\mathcal{H}}_{a b}$ function read $[17,18]$

$\tilde{\mathcal{H}}_{a b}^{(0)}\left(M^{2}, \mu_{R}^{2}\right)=\sigma_{a b}^{(0)}\left(M^{2}\right)$,

$\tilde{\mathcal{H}}_{a b}^{(1)}\left(M^{2}, \mu_{R}^{2}\right)=\sigma_{a b}^{(0)}\left(M^{2}\right)\left[\mathcal{A}_{0}+\frac{\pi^{2}}{6}\left(A_{a}^{(1)}+A_{b}^{(1)}\right)\right]$,

where the dependence on the renormalization scale $\mu_{R}$ of the infrared-finite part $\mathcal{A}_{0}$ of the renormalized virtual corrections $\sigma_{a b}^{V}$ is understood. The expression of the $\tilde{\mathcal{H}}_{a b}^{(1)}$ component is not general and assumes that the virtual contributions to the next-to-leading order cross section are normalized, in $4-2 \epsilon$ dimensions, as

$$
\begin{aligned}
\sigma_{a b}^{V}\left(M^{2}, \mu_{R}^{2}\right)= & \frac{\alpha_{s}}{2 \pi}\left(\frac{4 \pi \mu_{R}^{2}}{M^{2}}\right)^{\epsilon} \frac{\Gamma(1-\epsilon)}{\Gamma(1-2 \epsilon)} \sigma_{a b}^{(0)}\left(M^{2}\right) \\
& \times\left[\frac{\mathcal{A}_{-2}}{\epsilon^{2}}+\frac{\mathcal{A}_{-1}}{\epsilon}+\mathcal{A}_{0}\right]+\mathcal{O}(\epsilon) .
\end{aligned}
$$

It should be noted that for supersymmetric processes, these virtual corrections not only include pure QCD contributions, but also supersymmetric diagrams with squarks and gluinos running into the loops.

In order to avoid a double-counting of the logarithmic terms when combining the resummed cross section with the fixed-order result as given in Eq. (4), it is necessary to expand Eq. (7) at $\mathcal{O}\left(\alpha_{s}\right)$. The expanded partonic cross section is then given, in Mellin space, by $[17,18]$

$$
\begin{aligned}
\sigma_{a b}^{(\text {exp. })} & \left(N, M^{2}, \mu_{F}^{2}, \mu_{R}^{2}\right) \\
= & \tilde{\mathcal{H}}_{a b}^{(0)}\left(M^{2}, \mu_{R}^{2}\right) \\
& +\frac{\alpha_{s}}{2 \pi} \tilde{\mathcal{H}}_{a b}^{(1)}\left(M^{2}, \mu_{R}^{2}\right) \\
& -\frac{\alpha_{s}}{2 \pi} \ln \frac{\bar{N}^{2} \mu_{F}^{2}}{M^{2}} \sum_{c}\left[\gamma_{a c}^{(1)}(N) \tilde{\mathcal{H}}_{c b}^{(0)}\left(M^{2}, \mu_{R}^{2}\right)\right] \\
& -\frac{\alpha_{s}}{2 \pi} \ln \frac{\bar{N}^{2} \mu_{F}^{2}}{M^{2}} \sum_{c}\left[\tilde{\mathcal{H}}_{a c}^{(0)}\left(M^{2}, \mu_{R}^{2}\right) \gamma_{b c}^{(1)}(N)\right] \\
& -\frac{\alpha_{s}}{2 \pi} \tilde{\mathcal{H}}_{a b}^{(0)}\left(M^{2}, \mu_{R}^{2}\right)\left[\left(A_{a}^{(1)}+A_{b}^{(1)}\right) \ln { }^{2} \bar{N}\right] \\
& +\frac{\alpha_{s}}{\pi} \tilde{\mathcal{H}}_{a b}^{(0)}\left(M^{2}, \mu_{R}^{2}\right)\left[\left(\gamma_{a}^{(1)}+\gamma_{b}^{(1)}\right) \ln \bar{N}\right],
\end{aligned}
$$

the quantities $\gamma_{a b}^{(1)}$ being the full Mellin moments of the oneloop approximation of the Altarelli-Parisi splitting functions in four dimensions,

$\gamma_{q q}^{(1)}(N)=C_{F}\left[\frac{3}{2}+\frac{1}{N(N+1)}-2 \sum_{k=1}^{N} \frac{1}{k}\right]$

$\gamma_{g q}^{(1)}(N)=C_{F}\left[\frac{2+N+N^{2}}{N\left(N^{2}-1\right)}\right]$,

$\gamma_{q g}^{(1)}(N)=\tau_{R}\left[\frac{2+N+N^{2}}{N(N+1)(N+2)}\right]$,

$\gamma_{g g}^{(1)}(N)=2 C_{A}\left[\frac{1}{N(N-1)}+\frac{1}{(N+1)(N+2)}-\sum_{k=1}^{N} \frac{1}{k}\right]$

$+\beta_{0}$.

and $\gamma_{a}^{(1)}$ the field anomalous dimensions corresponding, in axial gauge, to the virtual, $N$-independent, pieces of $\gamma_{a a}^{(1)}[42]$

$\gamma_{q}^{(1)}=\frac{3 C_{F}}{2} \quad$ and $\quad \gamma_{g}^{(1)}=\beta_{0}$

\subsection{Transverse-momentum resummation at the next-to-leading logarithmic accuracy}

In order to be able to refactorize Eq. (2) without performing the integration over the transverse momentum $p_{T}$ and hence subsequently resum the large logarithmic contributions arising at small $p_{T}$, it is necessary to apply a Fourier transform to the partonic cross section $\sigma_{a b}$, 


$$
\begin{aligned}
\sigma_{a b}^{\text {(res.) }} & \left(N, M^{2}, p_{T}^{2}, \mu_{F}^{2}, \mu_{R}^{2}\right) \\
= & \int_{0}^{\infty} \mathrm{d} b \frac{b}{2} J_{0}\left(b p_{T}\right) \\
& \quad \times \sigma_{a b}^{\text {(res. })}\left(N, M^{2}, b^{2}, \mu_{F}^{2}, \mu_{R}^{2}\right),
\end{aligned}
$$

where $J_{0}(y)$ denotes the zeroth-order Bessel function. This operation renders the cross section explicitly dependent on the variable $b$, conjugate to the transverse momentum $p_{T}$, dubbed the impact parameter. This additional transformation allows us to correctly take into account transversemomentum conservation so that the partonic cross section can be rewritten under a form where soft and collinear radiation exponentiates [43-45],

$$
\begin{aligned}
\sigma_{a b}^{(\text {res. })} & \left(N, M^{2}, b^{2}, \mu_{F}^{2}, \mu_{R}^{2}\right) \\
= & \sum_{a^{\prime}, \tilde{a}, b^{\prime}, \tilde{b}} E_{a^{\prime} a}^{(1)}\left(N, 1 / \bar{b}^{2}, \mu_{F}^{2}\right) \\
& \times E_{b^{\prime} b}^{(1)}\left(N, 1 / \bar{b}^{2}, \mu_{F}^{2}\right) \mathcal{C}_{\tilde{a} a^{\prime}}\left(N, 1 / \bar{b}^{2}\right) \mathcal{C}_{\tilde{b} b^{\prime}}\left(N, 1 / \bar{b}^{2}\right) \\
& \times \mathcal{H}_{a^{\prime \prime} b^{\prime \prime}}\left(M^{2}, \mu_{R}^{2}\right) \exp \left[\mathcal{G}_{a^{\prime \prime} b^{\prime \prime}}\left(M^{2} \bar{b}^{2}, M^{2}, \mu_{R}^{2}\right)\right] .
\end{aligned}
$$

In this equation, holding at the next-to-leading logarithmic accuracy, the presence of the one-loop approximation of the QCD evolution operators allows for evaluating the parton densities at the natural scale of the process $1 / \bar{b}$, with $\bar{b} \equiv(b / 2) e^{\gamma_{E}}$. Moreover, all the other functions can be calculated perturbatively.

Although there are some freedoms, corresponding to the choice of a resummation scheme, in the way to separate the different contributions into the various $\mathcal{C}_{a b}, \mathcal{G}_{a b}$ and $\mathcal{H}_{a b}$ factors [46, 47], we adopt the most physical option where the Sudakov form factor and the $\mathcal{C}_{a b}$ function are free from any hard contribution. In this case, the Sudakov form factor is written as [23, 24]

$$
\begin{aligned}
\mathcal{G}_{a b}( & \left.M^{2} \bar{b}^{2}, M^{2}, \mu_{R}^{2}\right) \\
= & g_{a b}^{(1)}\left(\frac{\alpha_{s}}{2 \pi} \beta_{0} \ln \left[M^{2} \bar{b}^{2}\right]\right) \ln \left[M^{2} \bar{b}^{2}\right] \\
& +g_{a b}^{(2)}\left(\frac{\alpha_{s}}{2 \pi} \beta_{0} \ln \left[M^{2} \bar{b}\right], \frac{M^{2}}{\mu_{R}^{2}}\right),
\end{aligned}
$$

where the first term in this expansion,

$g_{a b}^{(1)}(\lambda)=\frac{1}{2 \lambda \beta_{0}}\left(A_{a}^{(1)}+A_{b}^{(1)}\right)[\lambda+\ln (1-\lambda)]$,

collects the leading logarithmic contributions, and the second term,

$$
\begin{aligned}
& g_{a b}^{(2)}\left(\lambda, M^{2} / \mu_{R}^{2}\right) \\
& \quad=\frac{1}{2 \beta_{0}}\left[B_{a}^{(1)}+B_{b}^{(1)}\right] \ln (1-\lambda)
\end{aligned}
$$

$$
\begin{aligned}
& +\frac{1}{2 \beta_{0}}\left[A_{a}^{(1)}+A_{b}^{(1)}\right]\left[\frac{\lambda}{1-\lambda}+\ln (1-\lambda)\right] \ln \frac{M^{2}}{\mu_{R}^{2}} \\
& +\frac{\beta_{1}}{2 \beta_{0}^{3}}\left[A_{a}^{(1)}+A_{b}^{(1)}\right]\left[\frac{\lambda+\ln (1-\lambda)}{1-\lambda}+\frac{1}{2} \ln ^{2}(1-\lambda)\right] \\
& -\frac{1}{2 \beta_{0}^{2}}\left[A_{a}^{(2)}+A_{b}^{(2)}\right]\left[\frac{\lambda}{1-\lambda}+\ln (1-\lambda)\right]
\end{aligned}
$$

the next-to-leading pieces. We recall that the relevant coefficients of the resummation functions $A_{a}$ and $B_{a}$ have already been introduced in Eq. (11) and Eq. (12).

In the 'physical' resummation scheme that we have adopted, the hard function $\mathcal{H}_{a b}$ is free from any logarithmic contribution and includes, as for threshold resummation, the finite parts of the renormalized virtual contributions $\mathcal{A}_{0}$ defined in Eq. (14). It reads, at the next-to-leading order accuracy $[23,24]$,

$\mathcal{H}_{a b}\left(M^{2}, \mu_{R}^{2}\right)=\sigma_{a b}^{(0)}\left(M^{2}\right)\left[1+\frac{\alpha_{s}}{2 \pi} \mathcal{A}_{0}\right]$.

Finally, the $\mathcal{C}_{a b}$ functions are evaluated, still at the next-toleading logarithmic accuracy and in this scheme, as [23, 24]

$\mathcal{C}_{a b}\left(N, \mu_{R}^{2}\right)=\delta_{a b}+\frac{\alpha_{s}}{2 \pi}\left[\frac{\pi^{2}}{6} C_{a} \delta_{a b}-\gamma_{a b}^{(1), \epsilon}(N)\right]$,

where $\gamma_{a b}^{(1), \epsilon}$ denotes the $\mathcal{O}(\epsilon)$ parts of the Altarelli-Parisi splitting kernels in Mellin space,

$$
\begin{aligned}
& \gamma_{q q}^{(1), \epsilon}(N)=\frac{-C_{F}}{N(N+1)}, \\
& \gamma_{q g}^{(1), \epsilon}(N)=\frac{-2 \tau_{R}}{(N+1)(N+2)}, \\
& \gamma_{g q}^{(1), \epsilon}(N)=\frac{-C_{F}}{N+1}, \\
& \gamma_{g g}^{(1), \epsilon}(N)=0 .
\end{aligned}
$$

After resumming the partonic cross section in the impact parameter $b$-space, the resummed cross section has to be transformed back to the physical $p_{T}$-space. This procedure requires to pay a particular attention to the singularities present in the resummed exponent when $\lambda=1$ in Eq. (21) and Eq. (22) that are related to the presence of the Landau pole in the perturbative running of the strong coupling constant. Following the prescription presented in Ref. [48], the inverse Fourier transform is calculated after deforming the integration contour of the $b$-integral into the complex plane by defining two integration branches

$b=e^{ \pm i \varphi} t \quad$ with $t \in\left[0, \infty[\quad\right.$ and $\quad \varphi \in] 0, \frac{\pi}{2}[$.

The Bessel function $J_{0}(y)$ appearing in Eq. (18) is then replaced by the sum of two auxiliary functions $h_{1}$ and $h_{2}$ that 
distinguish positive and negative phases in the complex $b$ plane,

$$
\begin{aligned}
& h_{1}(y, v)=-\frac{1}{2 \pi} \int_{-i v \pi}^{-\pi+i v \pi} \mathrm{d} \theta e^{-i y \sin \theta}, \\
& h_{2}(y, v)=-\frac{1}{2 \pi} \int_{\pi+i v \pi}^{-i v \pi} \mathrm{d} \theta e^{-i y \sin \theta} .
\end{aligned}
$$

For any choice of the $v$-parameter, these two functions are always finite and their sum is independent of $v$. This splitting has the advantage that each of the two functions is associated with only one single branch of the integration contour of Eq. (26).

In order to match with the fixed order result, making use of Eq. (4), the resummed cross section of Eq. (18), together with Eq. (19), we expand these two equations at $\mathcal{O}\left(\alpha_{s}\right)$,

$$
\begin{aligned}
\sigma_{a b}^{(\text {exp. })}\left(N, M^{2}, p_{T}^{2}, \mu_{F}^{2}, \mu_{R}^{2}\right) \\
=\mathcal{H}_{a b}^{(0)}\left(M^{2}, \mu^{2}\right) \\
+\frac{\alpha_{s}}{2 \pi} \mathcal{H}_{a b}^{(1)}\left(M^{2}, \mu^{2}\right) \\
\quad-\frac{\alpha_{s}}{2 \pi}\left[2 \mathcal{J}-\ln \frac{M^{2}}{\mu_{F}^{2}}\right] \sum_{c}\left[\mathcal{H}_{a c}^{(0)}\left(M^{2}, \mu_{R}^{2}\right) \gamma_{c b}^{(1)}(N)\right] \\
-\frac{\alpha_{s}}{2 \pi}\left[2 \mathcal{J}-\ln \frac{M^{2}}{\mu_{F}^{2}}\right] \sum_{c}\left[\gamma_{c a}^{(1)}(N) \mathcal{H}_{c b}^{(0)}\left(M^{2}, \mu_{R}^{2}\right)\right] \\
+\frac{\alpha_{s}}{2 \pi} \sum_{c}\left[\mathcal{H}_{a c}^{(0)}\left(M^{2}, \mu_{R}^{2}\right) \mathcal{C}_{c b}^{(1)}(N)\right] \\
+\frac{\alpha_{s}}{2 \pi} \sum_{c}\left[\mathcal{C}_{c a}^{(1)}(N) \mathcal{H}_{c b}^{(0)}\left(M^{2}, \mu_{R}^{2}\right)\right] \\
-\frac{\alpha_{s}}{8 \pi} \mathcal{H}_{a b}^{(0)}\left(M^{2}, \mu_{R}^{2}\right)\left[A_{a}^{(1)}+A_{b}^{(1)} i\right] \mathcal{J}^{2} \\
-\frac{\alpha_{s}}{4 \pi} \mathcal{H}_{a b}^{(0)}\left(M^{2}, \mu_{R}^{2}\right)\left[B_{a}^{(1)}+B_{b}^{(1)}\right] \mathcal{J} .
\end{aligned}
$$

We recall that the resummation coefficients appearing in this expression have already been introduced in Eq. (11) and Eq. (12) and that the Altarelli-Parisi splitting kernels have been presented in Eq. (16). Moreover, the first two coefficient of the perturbative expansion of the hard function $\mathcal{H}_{a b}$ and those of the function $\mathcal{C}_{a b}$ are deduced from Eq. (23) and Eq. (24). In addition, all the dependence on the transverse momentum has been embedded within the integral $\mathcal{J}$ defined by

$\mathcal{J}=\int_{0}^{\infty} \mathrm{d} b \frac{b}{2} J_{0}\left(b p_{T}\right) \ln \left[M^{2} \bar{b}^{2}\right]$.
2.4 Joint resummation at the next-to-leading logarithmic accuracy

In this section, we generalize the results of Sect. 2.3 so that both types of large logarithms arising either in the small $p_{T}$ region or near threshold are resummed simultaneously. Since these logarithms have the same dynamical origin, their joint reorganization is possible. In this way, they eventually exponentiate very similarly to the case of the transverse momentum regime of Eq. (19) [48-50],

$$
\begin{aligned}
\sigma_{a b}^{(\text {res. })} & \left(N, M^{2}, b^{2}, \mu_{F}^{2}, \mu_{R}^{2}\right) \\
= & \sum_{a^{\prime}, \tilde{a}, b^{\prime}, \tilde{b}} E_{a^{\prime} a}^{(1)}\left(N, M^{2} / \chi^{2}, \mu_{F}^{2}\right) \\
& \times E_{b^{\prime} b}^{(1)}\left(N, M^{2} / \chi^{2}, \mu_{F}^{2}\right) \mathcal{C}_{\tilde{a} a^{\prime}}\left(N, M^{2} / \chi^{2}\right) \\
& \times \mathcal{C}_{\tilde{b} b^{\prime}}\left(N, M^{2} / \chi^{2}\right) \\
& \times \mathcal{H}_{a^{\prime \prime} b^{\prime \prime}}\left(M^{2}, \mu_{R}^{2}\right) \exp \left[\mathcal{G}_{a^{\prime \prime} b^{\prime \prime}}\left(M^{2}, \bar{N}, \bar{b}, \mu_{R}^{2}\right)\right] .
\end{aligned}
$$

In order to ensure a proper refactorization of the cross section, a Fourier transform has again been performed, as in Eq. (18). Furthermore, we have introduced the function $\chi$, defined by

$\chi \equiv \chi(\bar{N}, \bar{b})=\frac{\bar{N}}{1+\bar{b} / \bar{N}}+\bar{b}$,

which interpolates between $\bar{N}$ in the threshold region, when $\bar{N} \gg \bar{b}$, and $\bar{b}$ in the small- $p_{T}$ region, when $\bar{b} \gg \bar{N}$. Even though there are several ways to define such an interpolation, the choice of Eq. (31) first implies that the leading and next-to-leading logarithms both in $\bar{b}$ and $\bar{N}$ are correctly reproduced, respectively in the limits $\bar{b} \rightarrow \infty$ and $\bar{N} \rightarrow \infty$. Next, it avoids the introduction of sizable subleading terms into perturbative expansions in $\alpha_{s}$ of the resummed formula of Eq. (30) that are not predicted by fixed-order computations.

While the $\mathcal{H}_{a b}$ and $\mathcal{C}_{a b}$ functions have exactly the same form as their counterparts in the small transversemomentum regime shown in Eq. (23) and Eq. (24), the Sudakov form factor now reads

$$
\begin{aligned}
\mathcal{G}_{a b}( & \left.M^{2}, \bar{N}, \bar{b}^{2}, \mu_{R}^{2}\right) \\
= & g_{a b}^{(1)}\left(\frac{\alpha_{s}}{2 \pi} \beta_{0} \ln \chi\right) \ln \chi \\
& \quad+g_{a b}^{(2)}\left(\frac{\alpha_{s}}{2 \pi} \beta_{0} \ln \chi, \ln \bar{N}, \frac{M^{2}}{\mu_{R}^{2}}\right),
\end{aligned}
$$

where the coefficients of its next-to-leading logarithmic accurate expansion are, in the $\overline{\mathrm{MS}}$-scheme, given by $[25,26]$ 


$$
\begin{aligned}
& g_{a b}^{(1)}(\lambda)=\frac{1}{2 \lambda \beta_{0}}\left(A_{a}^{(1)}+A_{b}^{(1)}\right)[2 \lambda+\ln (1-2 \lambda)], \\
& g_{a b}^{(2)}\left(\lambda, \ln \bar{N}, \frac{M^{2}}{\mu_{R}^{2}}\right) \\
& =-\frac{1}{\beta_{0}}\left[\gamma_{a}^{(1)}+\gamma_{b}^{(1)}\right] \ln (1-2 \lambda) \\
& -\frac{1}{2 \beta_{0}^{2}}\left[A_{a}^{(2)}+A_{b}^{(2)}\right]\left[2 \lambda \frac{1-\frac{\alpha_{s}}{\pi} \beta_{0} \ln \bar{N}}{1-2 \lambda}\right. \\
& +\ln (1-2 \lambda)] \\
& +\frac{1}{\beta_{0}}\left[A_{a}^{(1)}+A_{b}^{(1)}\right]\left[\lambda \frac{1-\frac{\alpha_{s}}{\pi} \beta_{0} \ln \bar{N}}{1-2 \lambda}\right. \\
& \left.+\frac{1}{2} \ln (1-2 \lambda)\right] \ln \frac{M^{2}}{\mu_{R}^{2}} \\
& +\frac{\beta_{1}}{2 \beta_{0}^{3}}\left[A_{a}^{(1)}+A_{b}^{(1)}\right] \\
& \times\left[\frac{(2 \lambda+\ln (1-2 \lambda))\left(1-\frac{\alpha_{s}}{\pi} \beta_{0} \ln \bar{N}\right)}{1-2 \lambda}\right] \\
& +\frac{\beta_{1}}{4 \beta_{0}^{3}}\left[A_{a}^{(1)}+A_{b}^{(1)}\right]\left[\ln ^{2}(1-2 \lambda)\right] \text {. }
\end{aligned}
$$

We recall that the resummation coefficients have already been shown in Eq. (11) and Eq. (12) and that the one-loop approximation of the field anomalous dimensions $\gamma_{a}^{(1)}$ are the $N$-independent parts of the Altarelli-Parisi splitting kernels (in axial gauge) given in Eq. (17).

Double counting implied when combining the resummed results presented above, after getting back to the physical $p_{T}$-space as shown in Sect. 2.3, is again removed by subtracting the expansion of Eq. (18), together with Eq. (30), at the first order in $\alpha_{s}$. This expansion has the same functional form as Eq. (28), after replacing the integral of the zerothorder Bessel function $\mathcal{J}$ by

$\tilde{\mathcal{J}}=\int_{0}^{\infty} \mathrm{d} b \frac{b}{2} J_{0}\left(b p_{T}\right) \ln \chi$.

\section{Installing and running RESUMMINO}

\subsection{Requirements and technical details}

In order to use RESUMMINO, several external libraries and header files are required and must be installed on the system.

First, information on the benchmark supersymmetric scenario under consideration is passed to the program by means of files compliant with the Supersymmetry Les Houches Accord (SLHA) conventions [51, 52]. We have adopted the choice to internally handle such files by making use of SLHAEA [53], a C++ header-only library dedicated to input, output and manipulation of SLHA data. While SLHAEA is fully included in RESUMMINO and hence does not need to be downloaded by the user, this tool relies on some headers of the Boos T C++ libraries [54] that are in contrast not provided with RESUMMINO. Therefore, their presence on the system is a necessary prerequisite and the two packages BOOST and BOOST-DEVEL must be available.

Next, both the fixed order and resummed components of the hadronic cross section require the evaluation of parton distribution functions, either in the physical $x$-space or in the conjugate Mellin $N$-space. The RESUMMINo package does not come with any built-in parton density fit and entirely relies on the external LHAPDF library [55] which must therefore be installed by the user. On run-time, the Mellin moment of the parton densities parameterization under consideration are obtained by a numerical fit performed by means of the Levenberg-Marquardt algorithm dedicated to multidimensional fits of non-linear functions [56, 57], as implemented in the GNU Scientific C++ Libraries (GSL). This algorithm consists of an iterative procedure using the method of least squares after a linearization of the fitting curves. As a consequence, both GSL header and library files must be installed by the user before being able to run RESUMMINO.

The knowledge of the parton densities both in $x$-space and $N$-space allows to compute all three components of the hadronic differential cross section $\mathrm{d}^{2} \sigma / \mathrm{d} p_{T} \mathrm{~d} M$ associated with the implemented physics processes, as described in Sect. 2. Additional integration upon the invariant mass $M$ of the gaugino or slepton pair or upon their transverse momentum $p_{T}$ then leads to the singly differential cross sections $\mathrm{d} \sigma / \mathrm{d} p_{T}$ and $\mathrm{d} \sigma / \mathrm{d} M$, respectively. Furthermore, integration upon both variables allows to extract total production rates. Let us note that in the case of threshold resummation, the integration upon $p_{T}$ has been performed analytically and there is no way to access doubly-differential cross sections. All these integrations, together with the usual two-body and three-body phase space integration relevant for the types of computations performed in RESUMMINO, are achieved by means of an adaptive multi-dimensional integration technique based on the importance sampling of the integration domain [58]. To this aim, we again make use of the GSL $\mathrm{C}++$ libraries provided with the adaptive multi-dimensional integration VEGAS algorithm [59].

Concerning the fixed order partonic cross sections, RESUMMINO is based on the leading order results of Ref. [19] and Refs. [20, 21] for slepton-pair and gaugino-pair production, respectively. Next-to-leading order corrections including both the QCD and supersymmetric QCD virtual diagrams are implemented as given in Ref. [17] and Ref. [18], the associated finite pieces of the virtual loops being computed by means of the QCDLOOP package [60]. The latter is 
fully embedded within RESUMMINO and thus does not need to be installed by the user.

\subsection{Installation}

We recommend the user to always use the latest stable version of RESUMMINO that can be downloaded from the webpage

http: / / www.resummino.org

Once downloaded, the package consists of a compressed tar file (resummino-x.x.x.tar.bz2 where $\mathrm{x}-\mathrm{x}-\mathrm{x}$ stands for the version number) that must be unpacked,

tar $\mathrm{xf}$ resummino-x.x.x.tar.bz2

In the case all the prerequisite dependencies of RESUMMINO are present on the system (see Sect. 3.1), it is then necessary to generate a Makefile appropriate to the system configuration. This is done by issuing in a shell the commands

cd resummino-x.x.x

cmake. [options]

The cmake program checks, in a first stage, that all the dependencies mandatory for RESUMMINO are correctly installed. It subsequently creates a series of Makefile scripts allowing for the compilation of the RESUMMINO source files and their linking with the dependencies. Two optional arguments can be passed to the cmake script. The first of these is related to the LHAPDF libraries. In the case they have not been installed in the directories referred to by the environment variable LD_LIBRARY_PATH (or DYLD_LIBRARY_PATH for MACOS systems), the LHAPDF installation directory must be specified by means of

\section{-DLHAPDF=/path/to/ lhapdf}

This instructs cmake that the LHAPDF libraries are stored in the directory /path/to/lhapdf / Iib and the header files in the directory/path/to/lhapdf/include. Equivalently, these two directories can be provided separately through the cmake options

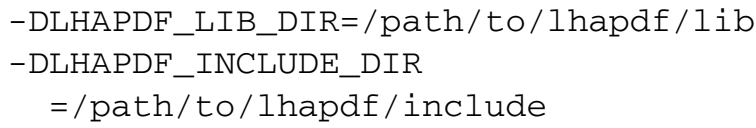

The second optional argument of the cmake script consists of information on the directory where the RESUMMINO executable has to be created (/path/to/install in the example below). This is specified by including the option
-DCMAKE_INSTALL_PREFIX=/path/to/install

when issuing the cmake command.

The Makefile can eventually be executed in order to generate a local release of RESUMMINO

make

make install

which can then be further used for physics applications.

\subsection{Running the code}

Once compiled, RESUMMINO can be immediately run from a shell by issuing

\section{resummino filename}

where filename consists of the path to a file containing the settings of the calculation to be performed. Three extra modes of running can also be employed by adding an optional flag when executing the code,

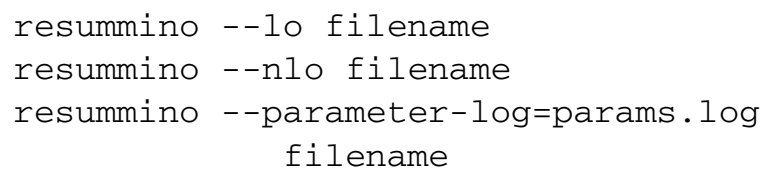

The first two choices above allow to respectively compute leading-order and next-to-leading order quantities (without matching to a resummation calculation). In contrast, the last of the three options leads to the generation of a file denoted by params. log that includes all the numerical values of the parameters defining the supersymmetric benchmark scenario under consideration.

Now, we turn to the way to encode the computation information in the input file to be parsed when executing the code. First, the definition of the collider is passed to the program by fixing the nature of the colliding beams and the hadronic center-of-mass energy (to be given in $\mathrm{GeV}$ ). This is achieved by configuring the variables collider_type and center_of_mass_energy, i.e., by including in the input file lines of the form of

collider_type = proton-proton

center_of_mass_energy $=8000$

Let us note that in the current version of the program, only proton-proton and proton-antiproton collisions are supported, so that the variable collider_type can only be set to one of the values proton-proton and proton-antiproton.

Next, the two produced superparticles must be referred to by setting the variables particle1 and particle2 
to the relevant Particle Data Group (PDG) codes [61]. We recall that since RESUMMINO is strictly dedicated to the production of the electroweak superpartners, only slepton, sneutrino, chargino and neutralino states are allowed as final state particles. For instance, the production of a lightest neutralino (whose the PDG code is 1000022) in association with a negatively-charged next-to-lightest chargino (whose the PDG code is -1000037) is encoded as

particle1 = 1000022

particle2 $=-1000037$

The numerical values of the masses of those particles, together with these of all the other supersymmetric model parameters, are provided by means of a file compliant with the SLHA conventions [51, 52], as already mentioned in Sect. 3.1. The path to this file is specified as the value of the variable slha,

slha $=$ slha.in

where in the example above, slha. in denotes a generic SLHA file.

The last pieces of information to be included in the input file define the type of computation to be performed and the numerical precision to be reached. The variable result allows to select the observable to compute by setting its value to total (total cross section $\sigma$ using the thresholdresummation formalism), pt (transverse-momentum distribution $\mathrm{d} \sigma / \mathrm{d} p_{T}$ using the $p_{T}$-resummation formalism), ptj (transverse-momentum distribution $\mathrm{d} \sigma / \mathrm{d} p_{T}$ using the joint-resummation formalism) or $\mathrm{m}$ (invariant-mass distribution $\mathrm{d} \sigma / \mathrm{d} M$ using the threshold-resummation formalism). For the last three possibilities, the numerical value of the transverse-momentum and the one of the invariant-mass at which the differential cross section must be respectively evaluated have to be referred to via the variables pt and M. For instance, implementing in the input file

result $=\mathrm{pt}$

pt $=50$

leads to the evaluation of $\mathrm{d} \sigma / \mathrm{d} p_{T}$ for $p_{T}=50 \mathrm{GeV}$, while

result $=\mathrm{M}$

$M=600$

implies the evaluation of $\mathrm{d} \sigma / \mathrm{d} M$ for $M=600 \mathrm{GeV}$. Furthermore, having instead

result $=$ total defines the computation of the total cross section. The parton density sets to be employed for both the leading-order and higher-order components of the calculated observable are indicated following the LHAPDF conventions which are based on an ordering according to parton density group names and numbers [55]. For instance, the command lines

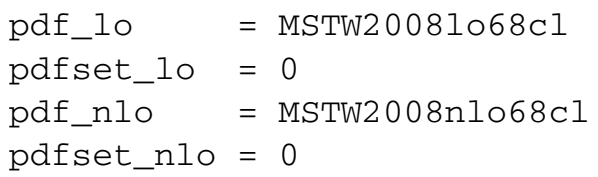

instruct RESUMMINO to use the best fits (the variables pdfset_lo and pdfset_nlo are set to zero) of the leading-order and next-to-leading-order fits of the MSTW 2008 parton densities [62] (as indicated by the variables pdf_lo and pdf_nlo). Factorization and renormalization scales are internally set to the sum of the mass of the produced particles, up to additional factors that must be specified in the input file via the intuitive variables mu_f and mu_r. For the sake of the example, central scale choices where scales are fixed to the average mass of the produced particles are enforced by

mu_f $=0.5$

mu_r $=0.5$

Finally, the speed of the computation is driven by two parameters, the numerical precision to be reached and the maximum of iterations allowed when VEGAS is numerically computing the various integrals presented in Sect. 2. These are related to two input variables, precision, which takes a real number as a value, and max_iter which refers to an integer number. Hence, including in the input file the lines

precision $=0.001$

max_iters $=3$

allows for three iterations of VEGAS and demands a relative precision of $0.1 \%$.

\section{Illustrative examples}

To illustrate the usage of RESUMMINO in a practical case, we perform several calculations in the framework of one representative constrained scenario of the Minimal Supersymmetric Standard Model (cMSSM). As designing an experimentally non-excluded supersymmetric scenario is going beyond the scope of this work, we refer to an earlier study performed by the LHC Physics Center at CERN together with both the ATLAS and CMS supersymmetry working groups [63]. This analysis is based on $1 \mathrm{fb}^{-1}$ of 
LHC data, electroweak precision observables and flavor physics constraints. Its conclusion consists of the proposal of several reference points in the cMSSM parameter space to be used for supersymmetric searches and phenomenological investigations. We adopt their 31 st scenario, where the ratio of the vacuum expectation values of the neutral components of the two Higgs doublets $\tan \beta$ is set to 40 and the Higgs supersymmetric mixing parameter $\mu$ is taken positive. At the supersymmetry-breaking scale, the universal scalar mass $m_{0}$ is fixed to $400 \mathrm{GeV}$, the universal gaugino mass $m_{1 / 2}$ to $600 \mathrm{GeV}$ and the universal trilinear coupling $A_{0}$ to $-500 \mathrm{GeV}$. After renormalization group running down to the electroweak scale, squarks and gluino are found heavy, with masses of about $1.5 \mathrm{TeV}$, with the exception of the lightest stop and sbottom states. The large leftright mixing inferred by the important negative value of $A_{0}$ indeed lowers their masses to $940 \mathrm{GeV}$ and $1100 \mathrm{GeV}$, respectively. In contrast, all the other superpartners (neutralinos, charginos, sleptons and sneutrinos) are lighter and lie in the $250-825 \mathrm{GeV}$ range. In the following, we further restrict ourselves to the production of the lightest electroweak superpartners whose masses are approximately given by

$$
\begin{aligned}
& m_{\tilde{\chi}_{1}^{0}}=250 \mathrm{GeV}, \quad m_{\tilde{\chi}_{2}^{0}}=m_{\tilde{\chi}_{1}^{ \pm}}=480 \mathrm{GeV}, \\
& m_{\tilde{e}_{L}}=m_{\tilde{\mu}_{L}}=565 \mathrm{GeV}, \quad m_{\tilde{e}_{R}}=m_{\tilde{\mu}_{R}}=460 \mathrm{GeV}, \\
& m_{\tilde{\tau}_{1}}=295 \mathrm{GeV}, \quad m_{\tilde{\tau}_{2}}=535 \mathrm{GeV} .
\end{aligned}
$$

Considering the LHC collider, running at a center-ofmass energy of $8 \mathrm{TeV}$, we focus in Table 1 on the largest total cross sections associated with the production of any pair of two of the particles under consideration. We indicate, in the

Table 1 Total cross sections associated with the production of any pair of superpartners among the lightest gauginos and sleptons ( $\tilde{\ell}$ equivalently denotes mass-degenerate selectrons and smuons) in the context of the LHC collider running at a center-of-mass energy of $8 \mathrm{TeV}$ and for the benchmark scenario 31 of Ref. [63]. The results are computed at the leading order (LO) and next-to-leading order (NLO) of perturbative QCD and then matched to threshold resummation at the next-toleading logarithmic accuracy (NLL+NLO). The corresponding scale uncertainties are also indicated and resummed cross sections smaller than $0.05 \mathrm{fb}$ are omitted

\begin{tabular}{llll}
\hline Final state & LO [fb] & NLO [fb] & NLO+NLL [fb] \\
\hline$\tilde{\chi}_{1}^{0} \tilde{\chi}_{1}^{0}$ & $0.1245_{-7.5 \%}^{+8.6 \%}$ & $0.1605_{-3.6 \%}^{+3.6 \%}$ & $0.1554_{-0.0 \%}^{+0.2 \%}$ \\
$\tilde{\chi}_{2}^{0} \tilde{\chi}_{2}^{0}$ & $0.0875_{-10 \%}^{+12 \%}$ & $0.1065_{-3.7 \%}^{+4.5 \%}$ & $0.1043_{-0.0 \%}^{+0.3 \%}$ \\
$\tilde{\chi}_{1}^{+} \tilde{\chi}_{2}^{0}$ & $4.3674_{-8.5 \%}^{+9.9 \%}$ & $4.8750_{-2.4 \%}^{+2.0 \%}$ & $4.8248_{-0.5 \%}^{+0.3 \%}$ \\
$\tilde{\chi}_{1}^{-} \tilde{\chi}_{2}^{0}$ & $1.4986_{-8.6 \%}^{+10 \%}$ & $1.7333_{-2.4 \%}^{+2.1 \%}$ & $1.7111_{-1.1 \%}^{+0.6 \%}$ \\
$\tilde{\chi}_{1}^{+} \tilde{\chi}_{1}^{-}$ & $2.8874_{-8.5 \%}^{+9.9 \%}$ & $3.3463_{-3.3 \%}^{+3.3 \%}$ & $3.3086_{-0.3 \%}^{+0.7 \%}$ \\
$\tilde{\ell}_{R}^{+} \tilde{\ell}_{R}^{-}$ & $0.0749_{-9.1 \%}^{+11 \%}$ & $0.0868_{-3.0 \%}^{+2.7 \%}$ & $0.0854_{-0.4 \%}^{+0.2 \%}$ \\
$\tilde{\ell}_{L}^{+} \tilde{\ell}_{L}^{-}$ & $0.0477_{-10 \%}^{+12 \%}$ & $0.0543_{-3.4 \%}^{+2.8 \%}$ & $0.0534_{-0.3 \%}^{+0.5 \%}$ \\
$\tilde{\tau}_{1}^{+} \tilde{\tau}_{1}^{-}$ & $0.5878_{-5.3 \%}^{+7.6 \%}$ & $0.7093_{-2.5 \%}^{+2.5 \%}$ & $0.6985_{-0.2 \%}^{+0.0 \%}$ \\
\hline
\end{tabular}

second column of the table, results at the leading-order of perturbative QCD, employing the leading order set of the 2008 MSTW parton densities [62]. In the third column, we compute next-to-leading order predictions, convolving the partonic cross section with the next-to-leading order set of the same parton density fit. Finally, in the fourth column, these last results are matched to threshold resummation. Although this does not imply a sensible change in the cross sections, we emphasize the importance of resummation by showing the theoretical uncertainties that are obtained after multiplying and dividing the central scale, set to the average mass of the produced particles (see Sect. 3.3), by a factor of two. Stabilization of the results can indeed be observed once soft and collinear radiation is resummed to all orders in $\alpha_{s}$. At the leading-order accuracy, the evolution of the parton densities introduces a dependence on the factorization scale through potentially large logarithmic terms, which leads to an uncertainty of about $\pm 10 \%$. Although this specific source of uncertainties is reduced at the next-to-leading order, new $\mathcal{O}\left(\alpha_{s}\right)$ diagrams imply an additional dependence on the renormalization scale. This yields a total scale uncertainty of a few percents. Finally, exponentiation, which allows to account for the dominant higher-order contributions within the Sudakov form factor, permits to render scale variations under a very good control, at the percent level.

In Fig. 1, we present transverse-momentum spectra related to the production of a selection of light chargino, neutralino and slepton pairs at the LHC. We first compute the predictions at $\mathcal{O}\left(\alpha_{s}\right)$ (dashed) and we next match the results with resummation in the transverse-momentum (dotted) and joint (plain) regimes. While the fixed-order results diverge at small transverse momentum due to the large logarithmic terms that have to be exponentiated, their resummation leads

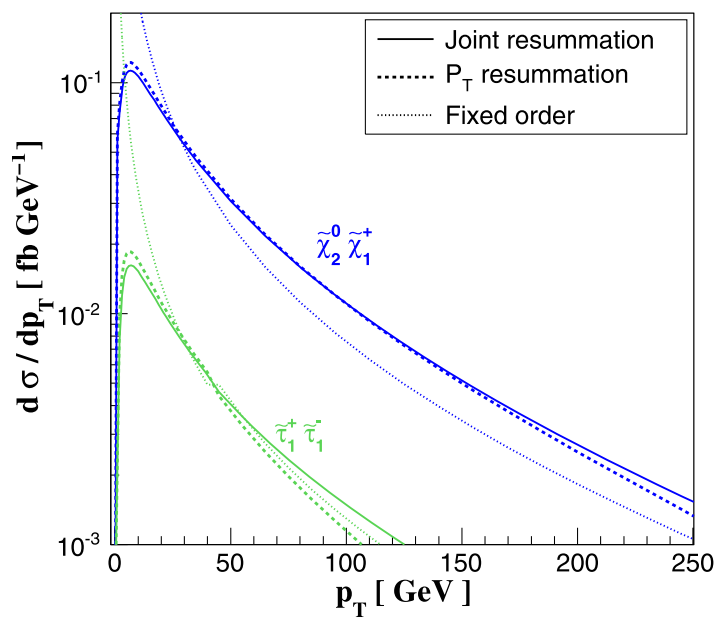

Fig. 1 Transverse-momentum distributions for $\tilde{\chi}_{2}^{0} \tilde{\chi}_{1}^{+}$and $\tilde{\tau}_{1}^{+} \tilde{\tau}_{1}^{-}$production at the LHC, running at a center-of-mass energy of $8 \mathrm{TeV}$, at order $\mathcal{O}\left(\alpha_{s}\right)$ (dashed) and after matching the results with transversemomentum (dotted) and joint (plain) resummation 
to a finite (and physical) behavior with a pronounced peak in the region where $p_{T} \lesssim 10 \mathrm{GeV}$. In this kinematical range, the asymptotic expansion of the two resummation formulas are in good agreement with the $\mathcal{O}\left(\alpha_{s}\right)$ results since they are all dominated by the logarithms. Therefore, matching with resummation as presented in Eq. (4) allows for the regularization of the next-to-leading order predictions for small values of $p_{T}$. The same matching effects also imply that the resummed predictions are sensibly larger than the fixed order ones when the transverse momentum of the superpartner pair lies within the intermediate $p_{T}$-range of $20-60 \mathrm{GeV}$. Finally, it is also shown that calculations using transversemomentum and joint resummation agrees reasonably well with each other on the entire $p_{T}$-range, although based on different Sudakov form factors.

\section{Summary}

In this paper, we have introduced the RESUMMINO package, a $\mathrm{C}++$ and FORTRAN program dedicated to precision calculations for gaugino and slepton pair production at hadron colliders. The program allows to compute total cross sections, invariant-mass and transverse-momentum distributions at leading order and next-to-leading order of perturbative QCD. In addition, the results are then matched to a resummation of the large logarithmic terms appearing at fixed order according to the transverse-momentum, threshold or joint resummation formalism.

We have illustrated the usage of our code by adopting a typical supersymmetric benchmark scenario for superpartner searches and performing in this context various computations by means of RESUMMINO. In the presented selection of results, we have chosen to emphasize the major advantages of making use of resummed predictions, i.e., a drastic reduction of the associated scale uncertainties and a regularization of the transverse-momentum spectrum in the small- $p_{T}$ region.

Acknowledgements This work has been supported by the BMBF Theorie-Verbund and by the Theory-LHC-France initiative of the CNRS/IN2P3.

Open Access This article is distributed under the terms of the Creative Commons Attribution License which permits any use, distribution, and reproduction in any medium, provided the original author(s) and the source are credited.

\section{References}

1. G. Aad et al., Phys. Lett. B 716, 1 (2012). doi:10.1016/j.physletb. 2012.08.020

2. S. Chatrchyan et al., Phys. Lett. B 716, 30 (2012). doi:10.1016/ j.physletb.2012.08.021
3. H.P. Nilles, Phys. Rep. 110, 1 (1984). doi:10.1016/03701573(84)90008-5

4. H.E. Haber, G.L. Kane, Phys. Rep. 117, 75 (1985). doi:10.1016/ 0370-1573(85)90051-1

5. https://twiki.cern.ch/twiki/bin/view/AtlasPublic

6. https://twiki.cern.ch/twiki/bin/view/CMSPublic/PhysicsResults

7. G. Aad et al., ATLAS-CONF-2012-154

8. S. Chatrchyan et al., CMS-PAS-SUS-12-022

9. A. Kulesza, L. Motyka, Phys. Rev. Lett. 102, 111802 (2009). doi:10.1103/PhysRevLett.102.111802

10. A. Kulesza, L. Motyka, Phys. Rev. D 80, 095004 (2009). doi:10.1103/PhysRevD.80.095004

11. W. Beenakker, S. Brensing, M. Kramer, A. Kulesza, E. Laenen et al., J. High Energy Phys. 0912, 041 (2009). doi:10.1088/ 1126-6708/2009/12/041

12. W. Beenakker, S. Brensing, M. Kramer, A. Kulesza, E. Laenen et al., J. High Energy Phys. 1008, 098 (2010). doi:10.1007/ JHEP08(2010)098

13. W. Beenakker, S. Brensing, M. Kramer, A. Kulesza, E. Laenen et al., Int. J. Mod. Phys. A 26, 2637 (2011). doi:10.1142/ S0217751X11053560

14. M. Kramer, A. Kulesza, R. van der Leeuw, M. Mangano, S. Padhi et al., arXiv:1206.2892 [hep-ph]

15. P. Falgari, C. Schwinn, C. Wever, J. High Energy Phys. 1206, 052 (2012). doi:10.1007/JHEP06(2012)052

16. W. Beenakker, M. Klasen, M. Kramer, T. Plehn, M. Spira et al., Phys. Rev. Lett. 83, 3780 (1999). doi:10.1103/PhysRevLett. 83.3780

17. G. Bozzi, B. Fuks, M. Klasen, Nucl. Phys. B 777, 157 (2007). doi:10.1016/j.nuclphysb.2007.03.052

18. J. Debove, B. Fuks, M. Klasen, Nucl. Phys. B 842, 51 (2011). doi:10.1016/j.nuclphysb.2010.08.016

19. G. Bozzi, B. Fuks, M. Klasen, Phys. Lett. B 609, 339 (2005). doi:10.1016/j.physletb.2005.01.060

20. G. Bozzi, B. Fuks, B. Herrmann, M. Klasen, Nucl. Phys. B 787, 1 (2007). doi:10.1016/j.nuclphysb.2007.05.031

21. J. Debove, B. Fuks, M. Klasen, Phys. Rev. D 78, 074020 (2008). doi:10.1103/PhysRevD.78.074020

22. B. Fuks, M. Klasen, D.R. Lamprea, M. Rothering, J. High Energy Phys. 1210, 081 (2012). doi:10.1007/JHEP10(2012)081

23. G. Bozzi, B. Fuks, M. Klasen, Phys. Rev. D 74, 015001 (2006). doi:10.1103/PhysRevD.74.015001

24. J. Debove, B. Fuks, M. Klasen, Phys. Lett. B 688, 208 (2010). doi:10.1016/j.physletb.2010.04.013

25. G. Bozzi, B. Fuks, M. Klasen, Nucl. Phys. B 794, 46 (2008). doi:10.1016/j.nuclphysb.2007.10.021

26. J. Debove, B. Fuks, M. Klasen, Nucl. Phys. B 849, 64 (2011). doi:10.1016/j.nuclphysb.2011.03.015

27. H. Contopanagos, G.F. Sterman, Nucl. Phys. B 419, 77 (1994). doi:10.1016/0550-3213(94)90358-1

28. S. Catani, M.L. Mangano, P. Nason, L. Trentadue, Nucl. Phys. B 478, 273 (1996). doi:10.1016/0550-3213(96)00399-9

29. G.F. Sterman, Nucl. Phys. B 281, 310 (1987). doi:10.1016/ 0550-3213(87)90258-6

30. S. Catani, L. Trentadue, Nucl. Phys. B 327, 323 (1989). doi:10. 1016/0550-3213(89)90273-3

31. S. Catani, L. Trentadue, Nucl. Phys. B 353, 183 (1991). doi:10.1016/0550-3213(91)90506-S

32. N. Kidonakis, G.F. Sterman, Nucl. Phys. B 505, 321 (1997). doi: 10.1016/S0550-3213(97)00506-3

33. N. Kidonakis, G. Oderda, G.F. Sterman, Nucl. Phys. B 525, 299 (1998). doi:10.1016/S0550-3213(98)00243-0

34. A. Vogt, Phys. Lett. B 497, 228 (2001). doi:10.1016/S03702693(00)01344-7

35. M. Kramer, E. Laenen, M. Spira, Nucl. Phys. B 511, 523 (1998). doi:10.1016/S0550-3213(97)00679-2 
36. S. Catani, D. de Florian, M. Grazzini, J. High Energy Phys. 0105, 025 (2001)

37. A. Kulesza, G.F. Sterman, W. Vogelsang, Phys. Rev. D 66, 014011 (2002). doi:10.1103/PhysRevD.66.014011

38. L.G. Almeida, G.F. Sterman, W. Vogelsang, Phys. Rev. D 80, 074016 (2009). doi:10.1103/PhysRevD.80.074016

39. W. Furmanski, R. Petronzio, Z. Phys. C 11, 293 (1982). doi: 10.1007/BF01578280

40. J. Kodaira, L. Trentadue, Phys. Lett. B 112, 66 (1982). doi:10. 1016/0370-2693(82)90907-8

41. S. Catani, E. D’Emilio, L. Trentadue, Phys. Lett. B 211, 335 (1988). doi:10.1016/0370-2693(88)90912-4

42. W.R. Frazer, J.F. Gunion, Phys. Rev. D 19, 2447 (1979). doi:10. 1103/PhysRevD.19.2447

43. J.C. Collins, D.E. Soper, Nucl. Phys. B 197, 446 (1982). doi:10. 1016/0550-3213(82)90453-9

44. J.C. Collins, D.E. Soper, Nucl. Phys. B 193, 381 (1981). doi:10. 1016/0550-3213(81)90339-4

45. J.C. Collins, D.E. Soper, G.F. Sterman, Nucl. Phys. B 250, 199 (1985). doi:10.1016/0550-3213(85)90479-1

46. S. Catani, D. de Florian, M. Grazzini, Nucl. Phys. B 596, 299 (2001). doi:10.1016/S0550-3213(00)00617-9

47. G. Bozzi, S. Catani, D. de Florian, M. Grazzini, Nucl. Phys. B 737, 73 (2006). doi:10.1016/j.nuclphysb.2005.12.022

48. E. Laenen, G.F. Sterman, W. Vogelsang, Phys. Rev. Lett. 84, 4296 (2000). doi:10.1103/PhysRevLett.84.4296
49. H.n. Li, Phys. Lett. B 454, 328 (1999). doi:10.1016/S03702693(99)00350-0

50. E. Laenen, G.F. Sterman, W. Vogelsang, Phys. Rev. D 63, 114018 (2001). doi:10.1103/PhysRevD.63.114018

51. P.Z. Skands, B. Allanach, H. Baer, C. Balazs, G. Belanger et al., J. High Energy Phys. 0407, 036 (2004). doi:10.1088/1126-6708/ 2004/07/036

52. B. Allanach, C. Balazs, G. Belanger, M. Bernhardt, F. Boudjema et al., Comput. Phys. Commun. 180, 8 (2009). doi:10.1016/ j.cpc.2008.08.004

53. http://fthomas.github.com/slhaea

54. http://www.boost.org

55. M. Whalley, D. Bourilkov, R. Group (2005)

56. K. Levenberg, Q. Appl. Math. 2, 164 (1944)

57. D. Marquardt, SIAM J. Appl. Math. 11, 431 (1963)

58. G.P. Lepage, J. Comput. Phys. 27, 192 (1978)

59. G.P. Lepage, Cornell preprint CLNS 80-447 (1980)

60. R.K. Ellis, G. Zanderighi, J. High Energy Phys. 0802, 002 (2008). doi:10.1088/1126-6708/2008/02/002

61. J. Beringer et al., Phys. Rev. D 86, 010001 (2012). doi:10.1103/ PhysRevD.86.010001

62. A. Martin, W. Stirling, R. Thorne, G. Watt, Eur. Phys. J. C 63, 189 (2009). doi:10.1140/epjc/s10052-009-1072-5

63. S. AbdusSalam, B. Allanach, H. Dreiner, J. Ellis, U. Ellwanger et al., Eur. Phys. J. C 71, 1835 (2011). doi:10.1140/epjc/ s10052-011-1835-7 